Industrial Control, Instrumentation and Signal Processing Track

\title{
SURFACE QUALITY CONTROL OF CERAMIC TILES USING NEURAL NETWORKS APPROACH
}

Contact author:

Dr.Željko Hocenski, assistant professor

Faculty of Electrical Engineering Osijek

Kneza Trpimira 2b

HR-31000 OSIJEK

Republic of Croatia

Phone: ++385 31224765

Mobile Phone: ++385 98373500

Fax: ++385 31208 905;

e-mail: zeljko.hocenski@etfos.hr

Emmanuel Karlo Nyarko, assistant

Faculty of Electrical Engineering Osijek

Kneza Trpimira 2b

HR-31000 OSIJEK

Republic of Croatia

Phone: ++385 31224750

Fax: ++385 31208905

e-mail: emmanuel.karlo.nyarko@etfos.hr 


\title{
SURFACE QUALITY CONTROL OF CERAMIC TILES USING NEURAL NETWORKS APPROACH
}

\author{
Željko Hocenski, Emmanuel Karlo Nyarko \\ JOSIP JURAJ STROSSMAYER UNIVERSITY OF OSIJEK \\ Faculty of Electrical Engineering Osijek
}

\begin{abstract}
:
The image processing described in this paper is used for visual quality control in ceramic tile production. The tiles surface quality depends on the surface defects. The described image processing is based on the neural network approach. The described diagnostic algorithm is presented to detect surface failures on white ceramic tiles. The tiles are scanned and the digital images are pre-processed and classified using neural networks. Pre-processing of the image data is used to keep the number of inputs of the neural networks performing the classification relatively small. It is important to reduce the amount of input data with problem specific pre-processing. The auto-associative neural network is used for feature generation and selection while the probabilistic neural network is used for classification. Simulation was performed in Matlab using the Neural Network Toolbox. The algorithm is evaluated experimentally using the real tile images. The analysis of the detection capabilities and sensitivity expressed in non-detected failures and false proclaimed defect is done also. The results obtained were satisfactory considering the fact that the images were scanned under the normal conditions. The developing and testing of this method is used for early design of the computer aided visual control.
\end{abstract}

Keywords: image processing, visual quality control, auto-associative neural networks, probabilistic neural networks, surface detection, ceramic tiles 


\section{INTRODUCTION}

The tiles surface quality is described by the surface defects and the intentional effects such as cracs, crazing, dry spots, pin hole, glaze devitrification, blister, etc. On-line testing of the surface defects is done at the tile factory by the visual testing performed by workers. This way of testing is liable to mistakes produced by human errors and subjectivity. Therefore the visual quality testing should be done more objectively by machine vision [1]. The image processing methods are developed for this purpose. Using the singular value decomposition (SVD) of the matrix of variations of the original image makes the image comparison duration shorter. The number of computer operations decreases by using SVD approximation by a lower rank matrix. But the too low rank of the matrix could yield bad comparison results [2].

The another method using the discrete wavelet transform - DWT was also used in failure detection [3]. To achieve robustness as well as good sensitivity of the algorithm, the images were divided into the segments. The analysis of detection capabilities is done for different segment sizes, different detection sensitivity levels - DSL and for two orthogonal wavelets. Furthermore, we investigated the influence of different wavelets to the diagnostic results and we found out that short wavelets are suitable for analysis (such as the Haar wavelet) and the optimal DSL depends on the wavelet too [4]. For the plain surfaces DSL has to be chosen depending on the visibility of the failure. The proposed algorithm could be used not just for detection but also for the classification of the tiles, so that our further research is planned to be carried out in that direction. [5][6]

So we tried to find the better method which is described in this paper. In image processing Neural Networks are used increasingly. A main application in this case is the classification of images. With the algorithm described here, errors on ceramic tile surfaces can be detected.

\section{BASICS OF NEURAL NETWORKS}

Neural Networks are based on replicating the functions and structure of the human/animal brain. Neural Networks are able to make decisions that are similar to human inference after a training period with several examples and the output desired for each example. They can be used for prognosis of complex situations, intelligent controlling or robot operation and recognition of object [7].

In this project an Auto-Associative Neural Network is used for feature generation and selection while a Probabilistic Neural Network is used for classification.

\section{NEURAL NETWORKS IN IMAGE PROCESSING AND CLASSIFICATION}

With the help of Neural Networks an error classification could be realised if the system is trained to make the correct decisions. For the use of Neural networks it is always important to have knowledge about the input data. In image processing, the enormous amount of data adds another problem for the use of Neural Networks. In the case of large networks, an enormous number of connections have to be handled. The number of connections controls the necessary amount of memory for the network and the calculation time and therefore the amount of data and the calculation time could be extremely high [8].

To keep the size of the network small, it is important to reduce the amount of input data with problem specific pre-processing. The pre-processing has to decrease the amount of input data but is not allowed to delete important information about the error. One possibility is to extract data out of the image that does not contain any image data. One example for this kind of pre- 
processing is the use of the Auto-Associative Neural Network. Such a network has $m$ input and $m$ output nodes and a single hidden layer with $n$ nodes with linear activations. During training the desired outputs are the same as the inputs. Such a network has a unique minimum and the outputs of the hidden layer constitute the projection of the input $m$-dimensional space onto an $n$-dimensional subspace [9].

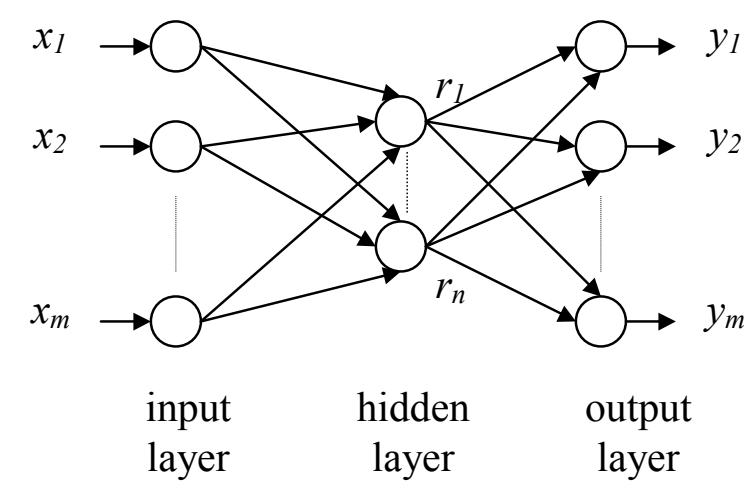

Fig. 1. Structure of an Auto-Associative Neural Network

The structure of the auto-associative net: where $x_{i}=y_{i}$. Hence the network is trained to produce an output equal its input.

The output from the middle (hidden) layer is then used as the input to the main neural network for the image to be classified. The Neural Network used for classification is the Probabilistic Neural Network. Such a network consists of 2 layers. When an input is presented, the first layer computes distances from the input vector to the training input vectors, and produces a vector whose elements indicate how close the input is to a training input. The second layer sums these contributions for each class of inputs to produce as its net output a vector of probabilities. Finally, a compete transfer function on the output of the second layer picks the maximum of these probabilities, and produces a 1 for that class and a 0 for the other classes.

\section{PROCESSING STEPS}

For the detection of errors and classification of images the processing steps implemented are shown in Figure 2. The grey scale digital images of the tiles are acquired (total set $=105$ images) using a colour scanner having a spatial resolution of $150 \mathrm{dpi}$. The image histogram is then obtained from the digital image. A set of Auto-Associative Neural Networks is then used to pre-process the data before it is fed to the Probabilistic Neural Network for classification.

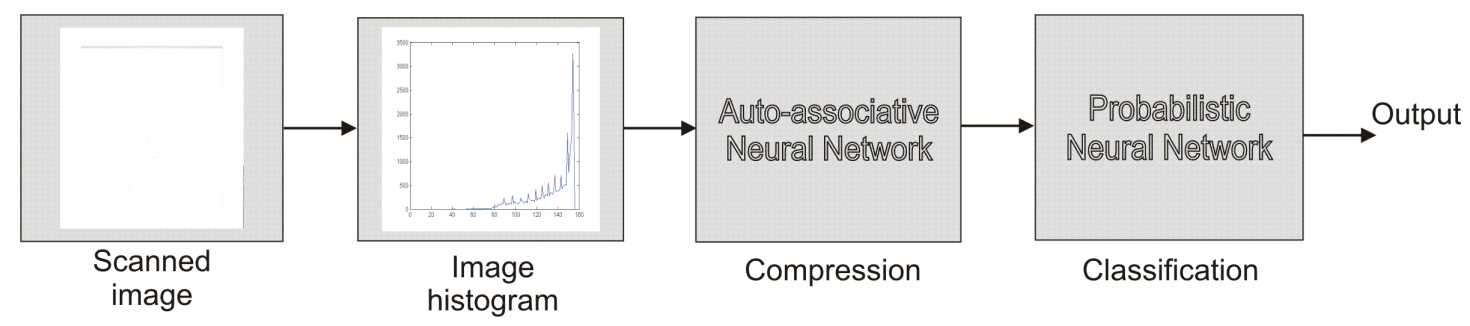

Fig. 2. Processing steps 
A detailed example is given below.

A typical digital image has a size of about $1200 \times 1200$ pixels. The image histogram of such an image is then created. This histogram, $\boldsymbol{H}$, is actually a vector with 256 components. This vector is then divided into 16 smaller vectors, $\boldsymbol{X}$, each of size 16 .

$\boldsymbol{H}=\left[\begin{array}{llllllll}h_{1} & h_{2} & \ldots & h_{16} & \ldots & h_{32} & \ldots & h_{256}\end{array}\right]$

The corresponding vectors are:

$\boldsymbol{X}_{1}=\left[\begin{array}{llll}h_{1} & h_{2} & \ldots & h_{16}\end{array}\right]$

$\boldsymbol{X}_{2}=\left[\begin{array}{llll}h_{17} & h_{18} & \ldots & h_{32}\end{array}\right]$

$\boldsymbol{X}_{16}=\left[\begin{array}{lllll}h_{241} & h_{241} & \ldots & h_{256}\end{array}\right]$

16 Auto-Associative Neural Networks then are trained one for each vector $\boldsymbol{X}_{\boldsymbol{i}}$. The corresponding vectors obtained from the hidden layers, $\boldsymbol{R}_{i}$, are used to form the input vector $\boldsymbol{R}$ for the Probabilistic Neural Network used in image classification.

$\boldsymbol{R}=\left[\begin{array}{llll}\boldsymbol{R}_{1} & \boldsymbol{R}_{2} & \ldots & \boldsymbol{R}_{16}\end{array}\right]$ where $\boldsymbol{R}_{i}=\left[\begin{array}{lll}r_{i} & r_{i+1}\end{array}\right], i=1 . .16$.

The Auto-Associative net always had 2 nodes in the hidden layer. The purpose of dividing the vector $\boldsymbol{H}$ and training a set of Auto-Associative nets instead of one neural network is to reduce memory requirements and computation time[10].

\section{RESULTS}

Simulation was performed in Matlab, using the Neural Network Toolbox. Looking at the histogram of a typical tile it was noticed that about the first 100 values (corresponding to intensity values 0 to 99) were zero. This was expected due to the fact that the tiles were white in colour and those intensity values correspond to the darker intensities on the grayscale. Results obtained using the whole histogram (256 values, 32 values when compressed) gave poor results. Using intensity values 97 to 256 (160 values, 20 values when compressed) gave better results but not satisfactory. Using values from 161 to 256 (96 values, 12 values when compressed) gave quite good results. However, using values from 100 to 195 (96 values, 12 values when compressed) gave the best results.

From 105 scanned tiles, 5 were good tiles and the remaining 100 tiles had some defect. The Auto-Associative Neural networks were trained using 3 of the good tiles chosen at random. 12 tiles were then chosen at random, 2 good and the remaining defective. These 12 tiles were used in training the Probabilistic Neural Network used in image classification. After the network was trained the results below in Table 1 were obtained when the whole set of tiles were tested. 
Table 1

\begin{tabular}{|l|c|c|}
\hline \multicolumn{1}{|c|}{ Tiles number } & Good Tiles & Defective Tiles \\
\hline $\begin{array}{l}\text { Number of tiles classified correctly } \\
\text { by the neural network (100-195) }\end{array}$ & 5 & 100 \\
\hline $\begin{array}{l}\text { Number of tiles classified correctly } \\
\text { by the neural network (161-256) }\end{array}$ & 4 & 87 \\
\hline $\begin{array}{l}\text { Number of tiles classified correctly } \\
\text { by the neural network (97-256) }\end{array}$ & 3 & 72 \\
\hline $\begin{array}{l}\text { Number of tiles classified correctly } \\
\text { by the neural network (1-256) }\end{array}$ & 2 & 63 \\
\hline
\end{tabular}

\section{CONCLUSION}

The image processing described in this paper is used for visual quality control in ceramic tile production. The visual quality testing is used to be more objectively by machine vision than the human eye. The described image processing is based on the neural network approach. The described diagnostic algorithm is presented to detect surface failures on white ceramic tiles. Pre-processing of the image data is used to keep the number of inputs of the neural networks performing the classification relatively small. The auto-associative neural network is used for feature generation and selection while the probabilistic neural network is used for classification. Simulation was performed in Matlab.

The algorithm is evaluated experimentally using the real tile images. The analysis of the detection capabilities and sensitivity expressed in non-detected failures and false proclaimed defect is done also. The tiles wrongly classified by the Probabilistic Neural Network had to be closely examined to prove that the classification was wrong. However a quick look by a human being almost always gave the same results.

The developing and testing of this method is used for early design of the computer aided visual control. It is necessary to continue the experiments with pattern tiles. It seems to be necessary to use a combination of several failure detection methods described and developed by authors to get the better results.

\section{REFERENCES}

[1]. R.Cucchiara, Cluster Analysis in Machine Vision with Genetic Algorithm, Machine Vision and Application Journal, Springer-Verlag, vol. 11, no. 1, 1998, pp. 1-8.

[2]. Ž.Hocenski, A.Baumgartner, Image Comparison Method for Visual Quality Control Based on Matrix Decomposition, IEEE International Symposium on Industrial Electronics, ISIE '2000, Universidad de las Américas-Puebla, México, 4-8 Dec.2000, Vol.2, pp.580-585

[3]. T.K. Sarkar, C.Su, R. Adve, M. Salazar-Palma, L. Garcia-Castillo, R.R. Boix, A tutorial on wavelets from electical engineering perspective, part1: Discrete wavelet techniques, IEEE Antennas and Propagation Magazine, vol.40,, no.5, Oct. 1998, pp. 49-68.

[4]. L. Gaudart, J. Crebassa, and J..P. Petrakian, Wavelet transform in human visual channels, Applied Optics,vol..32, no. 22, August 1993., pp. 4119-4127.

[5] Ž.Hocenski, S.Rimac-Drlje, T.Keser, Automatic Inspection of Defects in Plain and Texture Surfaces, IEEE International Conference on Intelligent Engineering Systems 2001, INES2001, Helsinki-Stockholm-Helsinki (Finland-Sweden), September 16-18, 2001, pp.221-227 
[6] Ž.Hocenski, S.Rimac-Drlje, T.Keser, Visual Diagnostics Based on Image Wavelet-Transform, $9^{\text {th }}$ European Conference on Power Electronics and Applications, EPE 2001, Graz, Austria, August 27-29, 2001, pp.627

[7] H.Vogel; U. Jäger; Error Detection on Structured Surfaces with Digital Image Processing and Neural Network; The European Congress on Intelligent Techniques and Soft Computing, (EUFIT), 1997.

[8] Simon Haykin; Neural Networks, A comprehensive Foundation; Prentice-Hall,Inc.,1999.

[9] Sergios Theodoridis; Konstantinos Koutroumbas; Pattern Recognition; Academic Press,1999.

[10] Vitorino Ramos; Pedro Pina; Fernando Muge; From Feature Extraction to Classification: A Multidisciplinary Approach applied to Portuguese Granites; Scandinavian Conference on Image Analysis,(SCIA), 1999. 